\title{
3) Application of Radioisotopes to the Diagnosis of Anemias
}

\author{
Gyoichi WaKisaKa, M. D. \\ Professor of Medicine, The First Division, Department of Internal \\ Medicine, Faculty of Medicine, Kyoto University, Kyoto
}

Radioisotopes are widely used for the diagnosis and elucidation of the pathogenesis of various anemias. In this paper the application of radioisotopes in this field was presented based on the experiences in our laboratory.

1) Studies of iron absorption by the simultaneous use of ${ }^{59} \mathrm{Fe}$ and ${ }^{55} \mathrm{Fe}$

A new analytical method for the study of iron absorption using ${ }^{59} \mathrm{Fe}$ and ${ }^{55} \mathrm{Fe}$ was described, and the absorption of iron from the intestinal tract was studied under various conditions. By this technique it was demonstrated that the absorption of iron from the intestine could be divided into two phases, namely the rapid phase and slow phase. In patients with iron deficiency anemia, the increase of iron absorption was mainly due to the increase of iron absorption in the rapid phase. There was a statistically significant correlation between the iron absorption in the rapid phase and the serum iron level as well as the plasma iron disappearance rate. It was confirmed that ferrous iron was more easily absorbed than ferric iron, and the absorption of iron was increased by the simultaneous oral administration of ascorbic acid or reduced glutathione.

2) Studies of iron metabolism in the reticuloendothelial system by the simultaneous use of ${ }^{59} \mathrm{Fe}$ and ${ }^{55} \mathrm{Fe}$

The functions of the reticuloendothelial system with regard to iron metabolism, namely the uptake of colloidal iron and the release of iron, were separately investigated using chondroitin sulfate iron labelled with ${ }^{59} \mathrm{Fe}$ and ${ }^{55} \mathrm{Fe}$ globulinate. In iron deficiency anemia, the release of iron from the reticuloendothelial system following the intravenous administration of colloidal iron was increased as compared with normal controls, while in rheumatoid arthritis and acquired hemolytic anemia, it was decreased as compared with normal controls. In aplastic anemia it was demonstrated that the release of iron from the reticuloendothelial system following the injection of colloidal iron was increased by the administration of steroid hormone.

3) Determination of the red cell life span in various diseases by the simultaneous use of ${ }^{51} \mathrm{Cr}$ and $\mathrm{DF}^{32} \mathrm{P}$

In normal controls, hemolytic anemia and aplastic anemia, the red cell life span measured by ${ }^{51} \mathrm{Cr}$ technique was parallel to that measured by $\mathrm{DF}^{32} \mathrm{P}$, while in iron deficiency anemia the red cell life span measured by ${ }^{51} \mathrm{Cr}$ technique was longer than that measured by $\mathrm{DF}^{32} \mathrm{P}$. This discrepancy was found to be due to the reduced elution rate of ${ }^{51} \mathrm{Cr}$ in iron deficiency anemia. In iron deficiency anemia there was 
statistically significant correlation between the red cell life span and the plasma iron disapperance rate, and the red cell life span tended to become normal following iron therapy.

4) Measurement of the function and size of the spleen by ${ }^{51} \mathrm{Cr}$ labelled red cells

Hemodynamics pattern of the spleen was studied in normal subjects and in patients with various anemias by analysing the splenic uptake curve and the dilution curve of red cell radioactivity in the peripheral blood following intravenous administration of ${ }^{51} \mathrm{Cr}$ labelled red cells. It was found that the hemodynamics pattern of the spleen could be divided into three components, A, B, and C. The rapidly mixing component $\mathrm{C}$, was supposed to represent the blood volume contained in the spleen, and was found to be markedly increased in congestive splenomegaly. The slow mixing component A was presumed to be related to the vascular space in the spleen, and was found to be increased in hereditary spherocytosis, acquired hemolytic anemia, iron deficiency anemia and congestive splenomegaly as compared with normal controls. There was a statistically significant correlation between the red cell life span and the size of the red cell pool in the spleen in patients with hereditary spherocytosis and congestive splenomegaly. It was also demonstrated that the scintigram of the spleen using ${ }^{51} \mathrm{Cr}$ labelled heated red cells or $\mathrm{MHP}-{ }^{197} \mathrm{Hg}$ or $\mathrm{MHP}-{ }^{203} \mathrm{Hg}$ was useful for detecting latent splenomegaly and measuring the volume of the spleen.

5) Studies of the absorption and metabolism of vitamin $B_{12}$ using radioactive $B_{12}$

The absorption test of vitamin $\mathrm{B}_{12}\left(\mathrm{~B}_{12}\right)$ and the studies of $\mathrm{B}_{12}$ metabolism using radioactive $B_{12}$ are very useful for the diagnosis and elucidating the pathogenesis of megaloblastic anemia in patients with pernicious anemia, total gastrectomy, blind loop syndrome and malabsorption syndrome. In recent years the whole body counter has become available for the studies of $B_{12}$ metabolism. The $B_{12}$ absorption test using the whole body counter has many advantages in simplicity and accuracy in contrast to such standard techniques as the fecal excretion test, urinary excretion test (Schilling) and hepatic uptake test.

It was found that the sliding method, in which the scintillation detector moves over the patient lying both in the supine and prone positions, was suitable for accurate measurement of the total radioactivity retained in the body irrespective of the difference in distribution of radioactive $B_{12}$. The result of $B_{12}$ absorption test obtained by the whole body counter coincided well with that obtained by the fecal excretion test. In 10 cases examined, the difference in the result of $B_{12}$ absorption test between the two methods was only $+0.7-1.1 \%$. The $B_{12}$ absorption as measured by the whole body counter was as follows: (mean \pm S. D.) normal subjects (10 cases) $75.5 \pm 9.2 \%$, partial gastrectomy (10 cases) $49.3 \pm 11.9 \%$, total or subtotal gastrectomy (6 cases) $1.9 \pm 1.6 \%$, pernicious anemia ( 1 case) $0.0 \%$, blind loop syndrome, (before treatment, 1 case) $1.9 \%$, (after treatment with antibiotics, 3 cases) $59.8 \pm 8.5 \%$. It was also found that the $B_{12}$ absorption in patients with gastric atrophy was also slightly decreased as compared with normal subjects. The high sensitivity of the 
whole body counter makes it possible to measure accurately the long-term turnover rates of $B_{12}$ over 1 to 3 years after the administration of radioactive $B_{12}$. In 2 normal subjects the biological half life of ${ }^{60} \mathrm{Co}-\mathrm{B}_{12}$ was 443 and 464 days, and the decay constant 0.156 and 0.149 percent loss per day, respectively, whereas in a patient with pernicious anemia and in a patient with total gastrectomy the biological half life of ${ }^{60} \mathrm{Co}_{0} \mathrm{~B}_{12}$ was 205 and 213 days and the decay constant 0.338 and 0.325 percent loss per day, respectively, indicating that the turnover rate of $B_{12}$ is increased in $\mathrm{B}_{12}$ deficiency. It was also demonstrated that it was possible to study the metabolism of two different forms of $\mathrm{B}_{12}$, such as cyanocobalamin, hydroxocobalamin or $B_{12}$ coenzyme, or two different states of $B_{12}$, such as free $B_{12}$ and intrinsic factor (IF) bound $B_{12}$, using the double isotope technique and the whole body counter. The detection of anti-IF antibody in the serum of patients with pernicious anemia using charcoal assay method and ${ }^{60} \mathrm{Co}-\mathrm{B}_{12}$ is useful for elucidating the pathogenesis of $\mathrm{B}_{12}$ deficiency.

6) Absorption test of folic acid using ${ }^{3} \mathrm{H}$-folic acid

The absorption test of folic acid (FA) using ${ }^{3} \mathrm{H}-\mathrm{FA}$ is important for the diagnosis of megaloblastic anemia due to FA deficiency. By measuring the urinary excretion of ${ }^{3} \mathrm{H}-\mathrm{FA}$ following the oral administration of ${ }^{3} \mathrm{H}-\mathrm{FA}$, it was possible to determine the intestinal absorption of $\mathrm{FA}$. In 11 normal subjects, the urinary excretion of ${ }^{3} \mathrm{H}$ FA during 24 hours following the oral administration of 10 or $20 \mu \mathrm{Ci}$ of ${ }^{3} \mathrm{H}-\mathrm{FA}$ together with $40 \mu \mathrm{g}$. per $\mathrm{Kg}$. body weight of cold FA was $48.4 \pm 15.0 \%$ (mean \pm 2 S.D.), while in patients with blind loop syndrome, intestinal resection and malabsorption syndrome the urinary excretion of ${ }^{3} \mathrm{H}-\mathrm{FA}$ was decreased as compared with normal controls, suggesting that the intestinal absorption of FA was impaired in these cases. 